\title{
Erratum: Conformal field theory of Painlevé VI
}

\section{O. Gamayun, ${ }^{a, b}$ N. lorgov $^{a, c}$ and O. Lisovyy ${ }^{a, c}$}

${ }^{a}$ Bogolyubov Institute for Theoretical Physics, 03680, Kyiv, Ukraine

${ }^{b}$ Physics Department, Lancaster University, Lancaster, LA1 4 YB, United Kingdom

${ }^{c}$ Laboratoire de Mathématiques et Physique Théorique CNRS/UMR 7350, Université de Tours, 37200 Tours, France

E-mail: o.gamayun@lancaster.ac.uk, iorgov@bitp.kiev.ua, lisovyi@lmpt. univ-tours.fr

ERRATUM TO: JHEP10(2012)038

Due to a typesetting error, ref. [21]:

A.V. Kitaev, Quadratic transformations for the sixth Painlevé equation, Lett. Math. Phys. 21 (1991) 105.

was inadvertently replaced by

B. Hanlon and G.C. Joshi, A noncommutative geometric approach to leftright weak interactions, Lett. Math. Phys. 27 (1993) 105 [hep-ph/9212209] [INSPIRE]. 